\title{
REGISTRO DEL PASO DE PEREGRINOS POR LAS RUTAS JACOBEAS ORENSANAS SEGUN LOS LIBROS PARROQUIALES
}

\author{
por \\ ENRIQUE BANDE RODRÍGUEZ
}

\section{CAMINOS ORENSANOS A SANTIAGO}

La provincia de Orense estuvo desde la plena y la baja Edad Media y lo sigue estando jalonada y vertebrada por los caminos que conducían a Santaigo. Por nuestra provincia pasaron y pasan caminos recorridos por peregrinos, comerciantes, pícaros, nobles, reyes, santos y fundadores de órdenes religiosas en peregrinación hacia la tumba del Apóstol. Las tierras orensanas no fueron nunca ajenas a los múltiples peregrinos que a lo largo de los siglos viajaron a Santiago ${ }^{1}$.

No se puede hablar de camino sino de caminos dado que las rutas a Compostela a su paso por la provincia de Orense fueron múltiples pero hubo dos rutas importantes que vamos a examinar con detención, estas son: El Camino Castilla, camino Oriental también llamado Vía de la Plata y los Caminos Portugueses. Los caminos Portugueses debieron ser muy importantes durante los siglos de la Reconquista dado que Portugal reconquistó antes que Castilla la zona que le había sido asignada en los tratados de Azmilra y Tudillén y por ello muchos peregrinos llegaron a Orense Ca-

${ }^{1}$ BANDE RODRÍGUEZ, E.: Caminos orensanos a Santiago. El Correo Gallego. 12Enero de 1992 y Arco Atlántico sexta feira Venres 22 de Janeiro de 1993.- Orense en el Jacobeo'93. La Región 20 - Febrero de 1992.

"CUADERNOS DE ESTUDIOS GALLEGOS", Tomo XLII, Fascículo 107, Santiago 1995. 
mino de Santiago por tierras portuguesas ya que las tierras castellanas estaban en lucha contra el Islam, aunque todos sabemos que la lucha de la Reconquista no fue nunca una guerra constante y encarnizada sino escaramuzas más o menos continuas con las que los musulmanes les recordaban a los reyes cristianos sus obligaciones, sus compromisos y sus pactos.

\section{El Camino de Castilla o Camino Oriental}

Aunque por la provincia de Orense pasaban varios Caminos a Santiago el más importante fue el de Castilla u Oriental o de la Plata. Este camino fue recorrido por peregrinos, comerciantes, y segadores que se dirigían a Castilla. En él confluían los peregrinos que por la "Ruta de la Plata" llegaban desde tierras andaluzas, desde Castilla, desde Portugal por Braganza, y desde el corazón de Europa. Este camino entraba en tierras orensanas por las Portillas del Padornelo y La Canda pasaba por Azibeiros, y Lubián para llegar después a Santa María de la Cabeza (Villavieja). Consultados los libros parroquiales de Santa María de Villavieja en uno de los difuntos que abarcaba desde el año 1648 a 1726 se nos dice que el 31 de Marzo de 1717 murió en la parroquia de Santa María de la Cabeza (Villavieja) Andrés Petrini de nacionalidad italiana quien pasaba peregrinando al Apóstol Santiago. Se nos dice en dicha partida que recibió los sacramentos y que se enterró de limosna con dos sacerdotes. La partida la firma Juan de Aparicio $^{2}$. En 1776 en Santa María de la Cabeza (Villavieja) en un libro sacramental que abarca desde 1722 a 1778 se nos dice que murió un peregrino del Apóstol Santiago de nacionalidad húngara según sus pasaportes, recibió el sacramento de la extremaunción y se enterró en esta iglesia. Firma la partida Antonio del Rio ${ }^{3}$.

De Villavieja el Camino continuaba por la parroquia de San Pedro de Pereiro. En el libro III de difuntos de la parroquia de San Pedro de Pereiro que abarca desde el año de 1789 al de 1853 figura una partida de defunción en la que se dice que murió de repente de una puñalada dada por un pere-grino que se dirigía a Compostela ${ }^{4}$.

\footnotetext{
${ }^{2}$ A.H.D.O.F.P. VILLAVIEJA, Santa María de la Cabeza I Bautizados y Difuntos. 1659-1722. 18.15.1

${ }^{3}$ A.H.D.O.F.P. VILlAVIEJA, Santa María de la Cabeza 2 Bautizados, Casados y Difuntos. 1722-1778. 18.15.2.

${ }^{4}$ A.H.D.O.F.P. PEREIRO, San Pedro 5 Difuntos. 1789-1853. 18.13.5.
}

"CUADERnOS DE ESTUdios GALlEGOS", Tomo XLII, Fascículo 107, Santiago 1995. 
En San Pedro de Pereiro confluía el Camino Portugués que venía de Braganza por Manzalvos, Cádavos, Mezquita, San Simón y Santigoso.

De Pereiro el Camino continuaba por Santa María del Cañizo, del paso de peregrinos por esta parroquia tenemos un testimonio que figura en el Libro de Bautizados, Casados y Difuntos que abarca desde el año de 1742 a 1824 donde se nos dice que el 24 de octubre del año 1788 murió un hombre que venía de Castilla enfermo y dijo que se llamaba Francisco Díaz y que era vecino del Rosal ${ }^{5}$. De Cañizo continuaba de la Villa de la Gudiña parroquia de San Pedro y San Martín, patria del beato Sebastián de Aparicio Apóstol de Méjico. De la parroquia de San Martín de la Gudiña en el libro 1 de Bautizos, Casados y Difuntos que abarca desde el año de 1680 al de 1825 tenemos un testimonio que nos dice que el año 1788 falleció un muchacho en la Venta de Espiño de la Sierra Seca, dijo que era de Pontevedra y que se llamaba Antonio Lorenzo, confeselo y no le pude administrar los sacramentos $\rangle^{6}$. En el libro de Bautizados, Casados y Difuntos que abarca desde el año de 1686 al de 1859 tenemos un testimonio que dice que el 16 de agosto de 1848 falleció y fue sepultado en el cementerio de San Martín de la Gudiña, José Sampedro natural de San Miguel de Espinoso, obispado de Orense, de trece años de edad que vivía de la siega ${ }^{7}$.

En la villa de la Gudiña apartaba un ramal, camino de herradura que por costumbres de las montañas divisorias de aguas de los ríos Camba y Mente, llegaba a Campobecerros, subía a Portocamba y por Eiras, Tez, Laza, Alberguería, Villar de Barrio, Bóveda y Junquera de Ambía y por Cantoña llegaba a Orense.

Ultimamente un investigador orensano basándose en la toponimia, da por cierto que dicho trazado por cumbres entre 900 y 1.000 metros de altitud donde se asientan las ventas de Espino, La Teresa, La Capilla, Bolaño y Juan Pérez es el que seguían los peregrinos convirtiendo así dicho ramal en un auténtico camino hasta la ciudad de Orense, después de pasar por Campobecerros, Laza, Alberguería, Junquera de Ambía, Pereiras, Venta del Río y Sejalvo donde hubo un hospital de peregrinos ${ }^{8}$.

${ }_{5}^{5}$ A.H.D.O.F.P. CAÑIZO, Santa María 1 Bautizados, Casados y Difuntos. 1742-1824. 18.16.1.

${ }^{6}$ A.H.D.O.F.P. GUDIÑA, San Martín 1 Bautizados, Casados y Difuntos. 1786-1825. Fol. 23. 18.5.1.

${ }^{7}$ A.H.D.O.F.P. GUDIÑA, San Martín 1 Bautizados, Casados y Difuntos. 1686-1859. 18.5.1.

${ }^{8}$ RIVAS QUINTAS, Eligio.: Camino Meridional de Santiago. Continuación de la Via de la Plata. Gráficas Folio S.L. Santiago 1993. Xunta de Galicia.

"CUADERNOS DE ESTUdIOS GALLEGOS", Tomo XLII, Fascículo 107, Santiago 1995. 
Reconozco que por allí hubo un camino pero se trata de un camino de arrieros, jalonado de ventas levantadas en el siglo XVIII, camino hoy abandonado. Era un camino de herradura, de gañanes, de pastores, de maleantes y de segadores que iban a Castilla procedentes de una zona muy concreta de la provincia de Orense 9 .

Continua el Camino por Erosa, San Lorenzo de Pentes como San Vicente de Navallo, San Lucas de Parada de la Sierra, Santiago de Carracedo, Santa María de Trepa y Fumaces. Testimonio fidedigno del paso del Camino son las Ventas de Juan Pérez, del Campo y de la Barrera de Orriós. Dicho Camino bajaba después a San Pedro de Osoño donde hubo un Hospital del que sólo queda el recuerdo de la toponimia dado que uno de los pueblos que componen esta parroquia lleva el nombre de Hospital. Del paso del camino por la parroquia de San Pedro de Osoño conservamos un testimonio en el libro II de Difuntos que abarca desde el año de 1785 al de 1851 en el folio 82 vuelto leemos que se le dio sepultura al cadáver de Jaceban Boyano natural de Godesal perteneciente a Benavente obispado de Astorga quien peregrinaba a Santiago, le mataron repentinamente y se le dio sepultura en la capilla de Osoño ${ }^{10}$.

En Osoño se le unían los peregrinos que venían de Portugal por Santa María de Vilarello da Cota, Santa Eulalia de Arzádegos, Santa Cruz de Terroso, San Juan de Enjames, Santa María de Vilar de Vos y San Miguel de Progo. Desde Osoño continuaba el Camino por Santa María de Abedes hasta Santa María de Verín, llamada en la Edad Media «Tierra de Varonceli» y según otros «Tierra de Viriato», sabemos que la Villa perteneció al monasterio de Celanova y que allí hubo un hospital de peregrinos y un lazareto. Verín fue cruce de caminos en las peregrinaciones jacobeas. Allí llegaba el Camino de Castilla que venía por Puebla de Sanabria, Padornelo y la Canda, Santa María de Villavieja, San Martín y San Pedro de la Gudiña. En Verín se unían a la vía castellana los peregrinos portugueses procedentes de Chaves, (Aquae Flaviae) sede episcopal y ciudad romana, y puerta de entrada a Galicia, Vilaverde da Raya, Santa María de Lama de Arcos, Santa María de Feces, Santa María de Mandín, Santa María de Tama-

\footnotetext{
${ }^{9}$ MADOZ, Pascual: Diccionario Geográfico-Estadístico de España y sus posesiones de ultramar. Tomo V. Madrid. 1846. pág. 370 (dicho diccionario hablando de Campobecerros dice «por dicho pueblo solo atraviesa un camino de "Herradura"».

${ }^{10}$ A.H.D.O. OSOÑO, San Pedro II Difuntos. 1758-1851. Fol. 82 V (Archivo Histórico Parroquial de Osoño) esta es la razón por la cual dicho libro no lleva signatura.
}

"CUADERNOS DE ESTUdiOS GALLEGOS", Tomo XLII, Fascículo 107, Santiago 1995. 
guelos, San Salvador de Cabreiroá. Cruzaban el valle del Támega por donde se desplazaban los comerciantes en dirección a Sanabraia ${ }^{11}$ y después de cruzar el Támega por el barrio de San Lázaro ascendían a Santa María de la Gracia (Monterrey) castillo almenado, situado en un cerro testigo donde hubo alberguería y tres hospitales de peregrinos, uno en el Colegio de la Compañía otro en el Colegio de San Francisco y otro en la Casa Colegio de San Juan Bautista. En 1763 llegaron al número de 1.900 los enfermos y murieron en los tres hospitales 900 los cuales fueron llevados en carros a enterrar. Los de la Compañía se enterraron en la huerta que llaman de Paez que es de Gerónimo y está por debajo del Colegio de Monterrey, y los de San Francisco se enterraron en la huerta de San Francisco a espaldas de la sacristía y de la enfermería ocho o nueve en cada hoyo. Fue tanta la epidemia que nadie quería asistir y por fuerza obligaron al obispo quien remitió cuatro capellanes a los hospitales por haberse puesto él malo. Esta noticia la firma Pedro Barreira Zambrano en $1763^{12}$.

Conservamos un testimonio claro y tajante del paso del Camino de Santiago por la Villa de Verín. El 28 de marzo de 1789 murió Diego Andrés natural del arzobispado de Toledo, recibió el sacramento de la penitencia y de la extremaunción, el de la eucaristía no pudo recibirlo. Venía de romería del Señor Apóstol Santiago y luego que llegó a esta Villa cayó postrado en tierra... No se pudo saber de que lugar determinado era. Enterrose en San Lázaro de limosna ${ }^{13}$.

Desde Monterrey un ramal del Camino iba por Santa María de Mixós, Santa Marina de Tetorta y San Juan de Laza donde enlazaban con el ramal que venía desde la Gudiña por Campobecerros, ramal que como ya vimos Madoz le llama «camino de herradura». El ramal principal o el Camino

${ }^{11}$ GONZÁLEZ CARNEIRO, Francisco: Chaves cidade Histórica. MCMLXXVIII. Braga, Portugal. (Verín fue cruce de Caminos en las Peregrinaciones Jacobeas en el Camino de Castilla, exclusivamente peninsular que venía por Puebla de Sanabria, Requejo, Padornelo, La Canda, Lubián y continuaba por Pereiro, Gudiña y Ventas hasta Verín para seguir por Ginzo y Allariz a Orense, Cea, Arenteiro, Lalín, y la Ulla. Pág. 129). Nos dice que la cita la toma TABOADA CHIVITE, Jesús: Guía de Monterrey Vigo. MCMLXXVIII. A Taboada Chivite le llama incansable investigador de Verín y Monterrey.

${ }^{12}$ A.H.D.O.F.P. MONTERREY, Santa María de la Gracia II. Bautizados, Casados y Difuntos. 1693-1883. Fol. 359. 27.11.2.

${ }^{13}$ A.H.P.V. (Archivo Parroquial de Verín) VERÍN, Santa María III Difuntos, 16761732 (dicho libro se conserva en el archivo parroquial de Santa María de Verín y por ello la cita no lleva signatura).

"CUADERNOS DE ESTUDIOS GALLEGOS", Tomo XLII, Fascículo 107, Santiago 1995. 
continuaba a Santiago de Albarellos. En Albarellos se le unían los peregrinos portugueses que venían por San Andrés de Rabal, San Ciprián, Santa Cruz, Santa María de Oimbra y San Salvador de Villaz; otros desde San Pedro de Quizanes cruzaban el Támega continuando hasta Albarellos por una ruta romana de la cual en la actualidad conservamos dos miliarios haciendo de mojones de deslindes en una viña en Pazos. Los peregrinos continuaban por San Martín de Rebordondo (Ruta testimoniada por los restos de miliarios encontrados) y por Santa María de Penaverde donde confluían los romeros que venían de Santa Eulalia de Bousés, Espiño, Santa María de Videferre, San Juan de la Granja, Santa María de Chas, San Salvador de Medeiros, San Pedro de Flariz, Boticas, Villar de Perdices, San Salvador de Vilamayor de la Gironda, San Pedro de Lucenza, San Salvador de Villar de Liebres, continuaba por Santa María de Lobaces, Santa Eulalia de Chamusiños, San Martín de Abavides y Santa Marina de Ginzo de Limia donde se le unían los peregrinos que venían por los Cotos Mixtos, San Juan de Randín y Santiago de Rubiás dos Mixtos. Después de cruzar el río Salas continuaba por Santa Eulalia de Maus de Salas y San Lorenzo de Tosende, el camino Portugués que venía de Montealegre continuaba por Santa María de Villamayor de Boullosa, San Bartolomé de Baltar, Santa María de Tejones, San Ciprián de Nocedo, Santiago de Garavelos y Santa María de Cima de Ribeira.

No faltan quienes desde lo alto de las Estivadas pretenden llevar el camino por las montañas que hay entre Santa María de Atanes y San Bartolomé de Baldriz hasta Santa María de la Alberguería a causa de la presencia de la laguna de Antela que con sus 26 kilómetros de largo y sus 7 de ancho impedía el paso del camino por Santa Marina de Ginzo de Limia. Sostiene que por la Villa de Ginzo de Limia nunca pasó camino alguno hasta que en plena euforia restauradora en tiempos de Sagasta fue construida la carretera nacional $525(\mathrm{~N}-525)$ cruzando la Laguna de Abavides a Sandianes ${ }^{14}$. Es cierto que la actual carretera es del siglo XIX pero antes hubo la vereda Real o Camino Real que en todo tiempo cruzó la Laguna de Antela por pontes o poldras.

Para mi esta claro que el Camino pasaba por Santa Marina de Ginzo de Limia, de ello conservamos un testimonio en el libro de Bautizados, Casados y Difuntos de la parroquia de Santa María de Ginzo de Limia, libro que

${ }^{14}$ JUSTO MÉNDEZ, Federico: Brotes de Raices históricas. Grupo Dos. Orense 1994. La Ruta de la Plata nunca pasó por Ginzo. La Región. 10 de junio de 1993.

"CUADERNOS DE ESTUdIOS GALLEGOS", Tomo XLII, Fascículo 107, Santiago 1995. 
abarca desde el año de 1661 al de 1746 , en una partida de dicho libro leemos que el 5 de mayo del año de 1670 falleció en esta Vila un hombre que venía de romería de Santiago de Compostela, no era muy alto de cuerpo, bien cargado. Hizo testamento delante de mi Juan Alonso y dijo que se llamaba... Natural de Aldreo Alcalde, arzobispo de Guarda en el Reino de Portugal. Dejó 15 tegas de pan para que las distribuyesen en esta Villa. Firmado Juan Rodríguez ${ }^{15}$.

Tenemos otro testimonio en el mismo Libro en el que nos dice que en diez y seis de octubre del año 1663 murió Amaro Fernández vecino del lugar de Otero jurisdicción de Segovia, Reino de Castilla, enterrose como pobre con asistencia de tres señores sacerdotes, no hizo testamento. Para que conste lo firma Cayetano de Villarino ${ }^{16}$. De que el Camino pasaba por Ginzo, aún conservamos otro testimonio en un Libro Parroquial de Santa María de Zos que abarca desde el año de 1731 al de 1806. En dicho libro se habla del Camino que iba a Ginzo así nos dice, en 25 de enero del año de 1780 se le dio sepultura a Benito Alonso vecino que fue del lugar dos Pardieiros feligresía de Lobaces cuyo cuerpo se había encontrado hecho cadáver en la mañana del día 4 en la «Vereda que viene de Ginzo hacia Abavides $\rangle^{17}$.

Siguiendo el Camino de Santiago en 1831 se inicia la apertura de la carretera de Vigo a Castilla sobre la vereda que desde Benavente se dirigía a Galicia por Puebla de Sanabria, Portillas, La Gudiña, Verín, Monterrey, Estivadas, Ginzo de Limia, Allariz y Orense ${ }^{18}$. El camino continuaba por Santa María de Couso de Limia donde se le unían los peregrinos que venían de Portugal por Lindoso, La Magdalena, San Salvador de Manín, Peneda Xerés, Portela do Home, Valle-Riocaldo Santa María, San Miguel de Lobios, Santa María de Cela antiguo monasterio dependiente de Osera, Santiagoso de Requiás, Santa María de Congostro, San Juan de Rairiz de Veiga, Santa María de Parada de Outeiro, San Juan de Villar de Santos,

${ }^{15}$ A.H.D.O.F.P. GINZO DE LIMIA, Santa Marina I. Bautizados, Casados y Difuntos. 1661-1746. 17.7.1.

${ }^{16}$ A.H.D.O.F.P. GINZO DE LIMIA, Santa Marina I Bautizados, Casados y Difuntos. 1661-1746. 17.7.1.

${ }^{17}$ A.H.D.O.F.P. ZOS, Santa María II, Bautizados, Casados y Difuntos. 1731-1806. Fol. $128 \mathrm{~V}$.

${ }^{18}$ NARDIZ ORTIZ, Carlos: El Territorio y las Comunicaciones en Galicia. Planos Históricos de la red viaria. Xunta de Galicia 1992.

"CUADERNOS DE ESTUdIOS GALLEGOS", Tomo XLII, Fascículo 107, Santiago 1995. 
Camino que seguía el trazado de la vía romana de Antonino llamada Via Nova o Vía XVIII, continuaba por las Poldras, y San Esteban de Sandianes. Del paso del Camino por San Esteban de Sandianes conservamos dos testimonios sacados de los libros parroquiales. En el Libro I de Difuntos de San Esteban de Sandianes leemos que el día 13 de mayo del año de 1750 se le dio sepultura en esta parroquia a Nicolás Ramirez pobre de solemnidad vecino de Santa María de Castrelo que murió de enfermedad natural viniendo de Monterrey, lo que indica que el Camino desde Monterrey seguía por Sandianes ${ }^{19}$. El otro testimonio nos dice que el 19 de noviembre de 1792 se le dió sepultura en esta parroquia a Manuel Laureiro soldado del regimiento de milicias de Betanzos, Compañía de Don Joseph Somoza, Arzobispado de Santiago, no recibió los sacramentos por haberse hallado muerto en la cama en mẹdio de sus compañeros cuando volvían de Chaves para Santiago ${ }^{20}$. El paso del Camino por Sandiás también esta documentado históricamente en un protocolo notarial que se conserva en el Archivo Histórico Provincial de Orense ${ }^{21}$.

Continuaba el Camino por San Juan de Piñeira de Arcos, Santiago de Coedo, San Salvador dos Penedos, Santiago de Allariz, Villa fundada por un Rey Suevo. La Villa de Allariz tuvo un hospital de peregrinos y un castillo derruido en la lucha de los Irmandiños, Villa que tiene tres iglesias románicas y un Claustro Gótico en el Monasterio de Santa Clara.

Del paso de peregrinos por la Villa de Allariz conservamos un testimonio claro y tajante en el libro de Bautizados, Casados y Difuntos en la parroquia de Santiago de Allariz que abarca desde el año de 1656 al de 1724. En dicho libro figura una partida de defunción que copiada a la letra dice:

${ }^{19}$ A.H.D.O.F.P. SANDIANES, San Esteban I Bautizados, Casados, Difuntos. 16001775. Fol. 325 V. 35.11.1.

${ }^{20}$ A.H.D.O.F.P. SANDIANES, San Esteban I Bautizados, Casados y Difuntos. 16001717. Fol. 340 V. 35.11.1.

${ }^{21}$ Testamento de un peregrino natural de Santa Olaya de Alexe quien con su mujer había salido hacía seis meses en romería a Nuestra Señora de Guadalupe, a Nuestra Señora de la Peña de Francia y de allí habían venido con el propósito de ir en romería al Apóstol Santiago de Galicia. Habiendo llegado a Villar de Santianes donde enfermó el otorgante y esta en cama muy malo punto de morir por lo que hace testamento el 28 de diciembre de 1598 (Protocolo de Melchor de Sandiás. Allariz Caixa 310 A.H.P.O. (Archivo Histórico Provincial de Orense).

"CUADERNOS DE ESTUDIOS GALLEGOS", Tomo XLII, Fascículo 107, Santiago 1995. 
«En diez y nueve días del mes de noviembre de mil seiscientos sesenta y seis años morió en el Hospital de esta Villa un peregrino que venía de Santiago en romería, con los sacramentos, decía que era de Madrid, enterrose de limosna. La Hermandad de San Lorenzo». Al margen dice: Un peregrino. Fol. 111.

Conservamos otros testimonios aunque menos elocuentes así en el libro de Bautizados, Casados y Difuntos de la parroquia de Santiago de Allariz que abarca desde el año 1730 al de 1816 se nos dice que falleció Pedro García, soltero vecino de San Esteban de Pezorres, jurisdicción del mismo nombre, en el obispado de Lugo, falleció en el tránsito que hacía del Reino de Portugal para su casa. Fol. 84.

En el mismo libro en el año de 1790 dice que le dio sepultura a Francisco Palomino vecino de la Villa de Madrid al tránsito que hacía por esta Villa. Fol. 88.

Desde la villa de Allariz el camino cruzaba el río Arnoya por un puente medieval, discurriendo después por Santiago de Folgoso, conservamos un testimonio en uno de los libros sacramentales donde leemos que el día 26 de noviembre del año de 1738 fue Dios servido de llevar para sí a un pobre que dijo venía de la romería de Nuestra Señora de las Ermitas y ser natural de Carballiño ${ }^{22}$.

De Folgoso el Camino iba a Santa Marina de Aguas Santas, uno de los santuarios gallegos más importantes después de la tumba del Apóstol Santiago en Compostela. Continuaba por Santiago de la Rábeda después de bajar pegado a los hornos de la Santa a la ermita de la Ascensión y al Castro de Armea por una antigua vía romana, continuaba el Camino por la venta del Río lugar donde se le unían los peregrinos que llegaban por Junquera de Ambía y Baños de Molgas. De aquí por la Castellana iba a San Verísimo de Sejalvo donde nos consta que hubo un hospital de peregrinos. De que el Camino pasaba por San Verisimo de Sejalvo conservamos un testimonio en un libro de difuntos del siglo XVI, hoy perdido, donde se nos dice que fallece una mujer de nacionalidad húngara que iba de romería a Santiago ${ }^{23}$.

${ }^{22}$ A.H.D.O.F.P. FOLGOSO, Santiago II Bautizados, Casados y Difuntos. 1674-1772. Fol. 136 V. 1.6.2.

${ }^{23}$ A.H.D.O.F.P. SEGALVO, San Verísimo I Bautizados, Casados y Difuntos. 16981782. Fol. 227. 30.20.1.

"CUADERNOS DE ESTUDIOS GALLEGOS", Tomo XLII, Fascículo 107, Santiago 1995. 
De Sejalvo el Camino continuaba a la ciudad de Orense por Mariñamansa donde se levantaba la ermita de Santa Eufemia y el Hospitalino y por la actual calle Bonhome penetraba en nuestra ciudad por la Porta da Aira, seguía por Fonte Arcada, Pena Vixía, Tandal da Figueira y la Plaza del Campo. En la ciudad de Orense los peregrinos se postraban a los pies del Santo Cristo «por acudir después a la Burgas fuentes salutíferas donde lababan sus pies llagados del duro bregar». Del paso del Camino por la ciudad de Orense conservamos testimonios en el libro del registro del Hospital de San Roque donde se nos dice que el 29 de noviembre de 1764 pasó por dicho Hospital el vecino de Morellada de Reis Benavente y el 9 de diciembre del mismo año pasó Juan Miguel Guberte de León ${ }^{24}$.

En otro libro de entrada y salida de enfermos que abarca desde noviembre de 1764 hasta 1801 encontramos refrencias a peregrinos que iban de camino a Santiago y eran atendidos en este centro ${ }^{25}$. En el artículo titulado «Hospitales de la Provincia de Orense» se nos dice que el 10 de marzo de 1638 fue bautizado un niño en la parroquia de la Santísima Trinidad, había nacido en el Hospital de Peregrinos y los padres eran dos romeros venidos de Ponferrada ${ }^{26}$. En el interrogatorio celebrado en Orense el 2 de julio de 1752, para confeccionar el Catastro de Ensenada cuando se trata de la pregunta 30 (Hospitales existentes en la ciudad), «los informantes digeron que en esta ciudad hay dos hospitales, el uno que llaman de San Roque extramuros de la misma... y otro que llaman Hospital de Peregrinos por que en él por tres días se permite descansar a los que pasan en Romería al Apóstol Santiago, los cuales no tienen existencia alguna sino un simple cubierto y paja para dormir». La administración corría a cargo de los abades de la Santísima Trinidad ${ }^{27}$. Conservamos otro testimonio en un libro de Difuntos de la Parroquia de la Santísima Trinidad de la Ciudad de Orense, que abarca desde el año de 1644 al de 1705, se nos dice que el día 20 de agosto de 1705 falleció Don Ignacio Da Silva el cual venía de Castilla y dijo ser hijo de Domingo Da Silva, vecino de la feligresía de Santa

\footnotetext{
${ }^{24}$ A.H.P.O. (Archivo Histórico Provincial de Orense) Libro de Registro del Hospital de San Roque. S. XVIII.

${ }^{25}$ A.H.P.O. HOSPITAL DE SAN ROQUE, (Caja No 5884).

${ }^{26}$ GALlego DOMÍNGUEZ, Olga: Hospitales de la Provincia de Orense. Bol. Auriense, Tomo VI, pág. 218.

${ }^{27}$ A.H.P.O. (Sign. 2564).
}

"CUADERNOS DE ESTUdiOS GALLEGOS", Tomo XLII, Fascículo 107, Santiago 1995. 
María de Agro, Arzobispado de Santiago, enterrose en la parroquia de la Santísima Trinidad ${ }^{28}$.

Aquí en la ciudad de Orense se le unían los peregrinos que venían desde Portugal por Braga, Viana do Castelo, Ponte Lima, Ponte de Barca, Lindoso, La Magdalena, San Salvador de Manín, parroquia hoy anegada por el embalse del Lindoso, Gusinde, Amenxueira, Santa María de Entrimo, San Martín de Grou, San Vicente de Lobera, San Miguel de Bangueses, Santa Eufemia de Milmanda, San Verísimo de Celanova, Puente Freixo, San Miguel de Espinoso, Alto de los Castros, Santa María de Pereiro de Montes, Santa María de Sobrado del Obispo, camino de Barbadás, La Barrera, La Plaza Mayor y la Catedral.

En la ciudad de Orense un ramal del camino cruzaba el río Miño por el Puente Viejo continuaba por el Pelegrín, Oira, El Veintiuno, San Pedro de Cudeiro, Souto, Costa, Sartédegos, Santiago de Gustey, continuaba por Venta Nova, Marco de San Esteban de Cambeo, Pontefechas, Santa María de Tamallacos, Bouzas, San Juan de Sobreira, Faramontaos, San Román de Viña, Cacifo, Pacio, San Miguel de Viduedo, Casas Novas, Paramios, Santos Facundo, y Primitivo de Cea. El otro ramal cruzaba el río Miño por el Puente Viejo, continuaba por Santiago de Caldas, San Miguel de Canedo, San Andrés de Castro de Beiro, Santiago de Parada de Amoeiro, Santa María de Louredo, Santa Eulalia de Pereda, continuando hasta la villa de Cea, después continuaba por el camino Real que llegaba hasta San Mamed de la Canda, San Juan de Barrar, donde se asienta el Santuario de la Peregrina, cruzaba el río Arenteiro y seguía por Santiago de Torrezuela, Santa María de Carballeda, Corna Santa María del Destierro para continuar a Castro Dozón ya en tierras de Pontevedra. Recientemente se ha publicado un libro que estudia el tramo del Camino desde la ciudad de Orense a Santiago $^{29}$, con el que no estamos muy de acuerdo en lo que dice de ese tramo del Camino a su paso por a las tierras Orensanas hasta las de la provincia de Pontevedra.

Nada mejor para rematar este apartado de la ponencia que recoger lo que nos dejó escrito Don Benigno González en la revista de la Parroquia y de la Escuela cuando nos dice:

${ }^{28}$ A.H.D.O.F.P. ORENSE, Santísima Trinidad, 29. Difuntos 1644-1705. Fol. 100 V. 30.16.29.

${ }^{29}$ REIMUNDEZ PORTELA, Manuel; ESPIÑO MATOS, José; SINDE LEMA, Mariano: El Camino de Santiago por el Sudeste. Orense Santiago de Compostela. Empuraginova, S.A. Santiago 1993.

"CUADERNOS DE ESTUdIOS GALLEGOS", Tomo XLII, Fascículo 107, Santiago 1995. 
«Los Caminos de los Peregrinos del sur, eran muy variados, pero todos venían a reunirse en Zamora y desde allí, por Puebla de Sanabria, atravesaban las Portillas y descendían al Valle de Monterey, desde donde por la Limia y Allariz llegaban a Orense y continuaban el viaje a Santiago por el mismo Camino que hoy sigue la actual carretera. Los portugueses gustaban más de viajar por las tierras de su reino, y desembocaban en la ciudad fronteriza de Chaves y desde allí entraban en el valle de Monterrey. $»^{30}$.

\section{Los Caminos Porgueses}

De Portugal salían hacia Orense dos Caminos importantes: Uno que entraba por Portela do Home y otro que entraba por Puente Barjas. Caminos por los que sabemos que llegaron a Santiago riadas de romeros y peregrinos.

El Camino Portugués por Portela do Home. Después de atravesar Peneda Xerés por Portela do Home seguía el trazado de la Vía XVIII de Antonino que iba de Braga a Astorga dicho Camino continuaba por Santa María del Valle de Riocaldo y San Miguel de Lobios, aquí confluían algunos de los peregrinos que entraban por la Magdalena y San Bento das Portas Abertas, dicho camino cruzaba el río por Ponte Pedriña, y continuaba por Porto Quintela. Un ramal por la baja Limia se dirigía a Sandiáns donde se unía con el camino oriental o de Castilla, otro ramal por Santa Comba de Bande, Santiago de Cadones donde se le unían los peregrinos que venían de Portugal por Castro Leboreiro, continuaba por San Lorenzo de Cañón, donde se halló un miliario, San Verísimo de Celanova, donde se le unían algunos peregrinos que llegaban por San Miguel de Bangueses, Santa Eufemia del Milmanda y Santa María de Castromao, el Camino continuaba por San Salvador de Vilanova dos Infantes, Santa María de Puente Fechas, Santo Tomé de Barja, donde se encontró un miliario en Barxiña continuaba a la ciudad de Orense por la Farixa donde se halló un miliario en la antigua capilla dedicada a San Juan hoy derruida, continuaba por el Polvorín y entraba en la ciudad de Orense por la Puerta del Villar para después cruzar el río Miño por los lugares ya antes apuntados.

${ }^{30}$ GONZÁLEZ SOLOGAISTUA, Benigno: Influencia Económica de las Peregrinaciones a Compostela. Biblioteca de la «Misión», Revista de la Parroquia y la Escuela. Suplemento de «Misión» N 52, pág. 8. 31.7.1937.

"CUADERNOS DE ESTUDIOS GALLEGOS", Tomo XLII, Fascículo 107, Santiago 1995. 
El Camino Portugués por Puente Barja. Este camino venía de Portugal por Melgazo, San Gregorio y continuaba por San Cipriano de Padrenda, San Miguel de Desteriz, Santa María de Condado (donde tenemos documentado un Hospital de Peregrinos), San Martín de Valongo, Santiago de Cortegada, San Salvador de Arnoya donde se le unían los peregrinos que venían por San Juan de Monteredondo, San Juan de Crespos, San Pedro de la Torre, San Pedro y Santa María de Leirado, Santa María de Pao, San Pedro de Poulo, Santa María de Zaparín. Desde la Arnoya continuaba a la Villa de Ribadavia, después de cruzar en barcas el río Miño. Aquí se bifurcaban en dos ramales:

Uno se dirigía a Avión por San Cristóbal de Regodegón, San Miguel de Carballeda, San Andrés de Abelenda das Penas, Santa María de Beade, San Mauro de Regadas, San Verísimo de Verán, San Miguel de Lebosende, Santo Tomé de Serantes, Santa María de Lamas, otros cruzaban el río Avia para continuar por San Salvador de Pazos de Arenteiro, San Martín de Cameija, San Julián de Astureses, Santiago de Corneda, San Pedro de Dadín, hasta Castro do Zon, donde se unía otro ramal desde Ribadavia iba por Santa María de Esposende, Santa María de Gomariz, San Clodio de Ribero, Santiago de Anllo, Santa María de Grixoa, Santiago de Partovia, La Villa de Carballiño, Santiago de Murelos, San Miguel de Piteira, y Santa Eugenia de Lobanes para unirse al camino de Castilla en el Arenteiro.

El impacto del Camino debió ser una de las causas de que la Orden de Santiago tuviese en nuestra diócesis las parroquias de:

Veiga, San Munio en la Bola, Campobecerros, Santiago y su anejo Portocamba, San Miguel en Castrelo del Valle, Barra, Santa María y su anejo Ucelle en Coles, Garabanes, San Pedro en Maside, Carracedo, Santiago en la Peroja, Codosedo, Santa María y su anejo Paradiña, Santa María Magdalena en Villar de Barrio, Villar de Santos San Juan, llamada también de San Juan de Sáa. Tenía además en Orense la Encomienda de la Batundeira. 